\title{
Características epidemiológicas y clínicas de las onicomicosis causadas por Fusarium spp. en un centro de referencia de Bogotá, Colombia, 2001-2010
}

Epidemiological and clinical features of onychomycosis caused by Fusarium spp. in a reference center from Bogotá, Colombia, 2001-2010

\author{
Camilo Andrés Morales ${ }^{1}$, Andrea Solórzano², Aída Paola Rojas ${ }^{3}$ \\ 1. Médico dermatólogo, Oficina de Docencia e Investigación, Centro Dermatológico Federico Lleras Acosta, E.S.E., Bogotá, D.C., Colombia; \\ instructor, Facultad de Medicina, Universidad CES, Medellín, Colombia \\ 2. Médica, residente de tercer año de Dermatología, Facultad de Medicina, Universidad CES, Medellín, Colombia; Centro Dermatológico Federico \\ Lleras Acosta, E.S.E., Bogotá, D.C., Colombia \\ 3. Médica epidemióloga, Oficina de Docencia e Investigación, Centro Dermatológico Federico Lleras Acosta, E.S.E., Bogotá, D.C., Colombia; \\ instructora, Facultad de Medicina, Universidad CES, Medellín, Colombia
}

\section{Resumen}

INTRODUCCión. Fusarium spp. es uno de los agentes etiológicos de onicomicosis por mohos no dermatofitos aislados con mayor frecuencia en todo el mundo. Sin embargo, la información disponible sobre el tema es limitada y algunas veces contradictoria, probablemente debido al escaso número de casos informados. El objetivo del estudio fue describir las características epidemiológicas y clínicas de un grupo de pacientes con diagnóstico confirmado de onicomicosis por Fusarium spp. en un centro de referencia de Bogotá, Colombia.

MATERIALES Y MÉTodos. Se llevó a cabo un estudio descriptivo de tipo corte transversal, en el que se describieron las características epidemiológicas y clínicas de los pacientes con diagnóstico confirmado de onicomicosis por $\mathrm{Fu}$ sarium spp. que consultaron entre el $1^{\circ}$ de enero de 2001 y el 31 de diciembre de 2010.

RESULTADOS. Se confirmaron 95 casos de onicomicosis por Fusarium spp. en 72 mujeres ( $76 \%$ ) y 23 hombres ( $24 \%$ ), con una edad promedio de 40 años. Ocho $(8,4 \%)$ tenían insuficiencia vascular, seis $(6,3 \%)$ hipotiroidismo, y dos $(2,1 \%)$ diabetes. Las presentaciones clínicas más frecuentes fueron la onicomicosis subungular distal y lateral en 71 pacientes $(74,7 \%)$ y la distrófica total en 10 pacientes $(10,5 \%)$. Se presentó un caso de infección mixta con Neoscytalidium dimidiatum.

ConClusiones. La presentación clínica más frecuente de las onicomicosis por Fusarium spp. es la subungular distal y lateral, con cromoniquia de tonos blanco y amarillo, que puede ser indistinguible de las onicomicosis causadas por dermatofitos o por otros mohos. No se encontró relación con factores de riesgo que pueden predisponer a la infección.

PALABRAS ClAVE: onicomicosis, mohos, Fusarium, epidemiología.

\section{Summary}

INTRODUCTION: Fusarium spp. is one of the etiological agents of onychomycosis by non dermatophytic molds most frequently isolated worldwide. However, the available information on the subject is limited and sometimes contradictory,

\section{Correspondencia:}

Camilo Andrés Morales

Email:

camiderm@yahoo.com

Recibido: 20 de octubre de 2012. Aceptado: 15 de diciembre de 2012.

No se reportan conflictos de intereses. 
probably due to the small number of reported cases. The aim of the study was to describe the epidemiological and clinical features of a group of patients with confirmed onychomycosis due to Fusarium spp. in a referral center from Bogotá, Colombia.

METHODS: A cross-sectional descriptive study type was conducted on patients with confirmed diagnosis of onychomycosis by Fusarium spp. who consulted between January $1^{\text {st }}$, 2001, and December $31^{\text {st }}$, 2010, to describe its epidemiological and clinical features.

RESUlTS: We confirmed 95 cases of onychomycosis by Fusarium spp. in 72 women $(76 \%)$ and 23 men ( $24 \%)$, with an average age of 40 years. Eight $(8.4 \%)$ had vascular insufficiency, six (6.3\%) hypothyroidism, and two (2.1\%) diabetes. The most frequent clinical presentations were distal and lateral subungual onychomycosis in 71 patients $(74.7 \%)$ and total dystrophic onychomycosis in 10 patients $(10.5 \%)$. One case of mixed infection with Neoscytalidium dimidiatum was observed.

CoNCLUSION: The most common clinical presentation of onychomycosis by Fusarium spp. is the distal and lateral subungual, with leukonychia or yellow decoloration, which may be indistinguishable from onychomycosis caused by dermatophyte or other molds. No relationship was found with risk factors that may predispose to infection.

KEY WORDS: Onychomycosis, molds, Fusarium, epidemiology.

\section{Introducción}

Los mohos no dermatofitos son hongos filamentosos de distribución universal, saprófitos de los suelos y fitopatógenos habituales ${ }^{1-3}$. Aunque tradicionalmente fueron considerados agentes contaminantes y patógenos secundarios de las uñas distróficas o colonizadas por dermatofitos debido a su virtual incapacidad para invadir tejidos queratinizados ${ }^{4-6}$, en la actualidad son una causa importante de lesiones cutáneas y onicomicosis, con un incremento progresivo en el número de casos en diferentes países ${ }^{7-9}$ a partir de los primeros aislamientos informados en la década de los años $70^{10-12}$.

La taxonomía de los mohos del género Fusarium es compleja debido a la gran cantidad de especies que se encuentran en la naturaleza ${ }^{13,14}$, de las cuales, $F$. chlamydosporum, F. dimerum, F. incarnatum, F. moniliforme, F. nivale, F. oxysporum, F. proliferatum, F. sacchari, F. solani, F. tabacinum y F. verticillioides son los principales patógenos humanos ${ }^{15,16}$. Fusarium solani y F. oxysporum son las especies que más se han relacionado con onicomicosis ${ }^{13,15,17,18} \mathrm{y}$ con problemas fitosanitarios de gran impacto en la agricultura y la floricultura colombianas ${ }^{19-22}$, principalmente, en la producción de claveles $^{20,23}$. Recientemente se ha sugerido que estos cultivos son un potencial reservorio de especies de Fusarium patógenas para el ser humano ${ }^{20}$.

Como cualquier otro moho no dermatofito, Fusarium spp. puede comportarse como contaminante ambiental, o colonizador transitorio o permanente de las uñas distróficas ${ }^{1,24}$, complicar una tinea unguium ${ }^{6,24}$ o ser agente etiológico de onicomicosis en individuos previamente sanos $^{1,16}$. Fusarium solani tiene la capacidad de degradar la queratina ${ }^{13,25}$ y diferentes especies de Fusarium producen micotoxinas y proteasas con propiedades similares a las de la tripsina y la subtilisina, potencialmente tóxicas para la epidermis y la dermis ${ }^{2,25}$; sin embargo, aún se desconocen los mecanismos por medio de los cuales el moho invade y penetra el estrato córneo.

Fusarium spp. es el agente etiológico de onicomicosis por mohos no dermatofitos aislado con mayor frecuencia en Colombia ${ }^{7,26-29}$; correspondió a $50 \%$ de los aislamientos de mohos no dermatofitos en una serie de $310 \operatorname{casos}^{27}$ y existe un significativo número de pacientes afectados en diferentes ciudades del país (TABLA 1).

Algunos autores han sugerido que las onicomicosis causadas por este moho pueden tener relación con factores de riesgo particulares, como los oficios relacionados con la agricultura y la jardinería ${ }^{18,30}$, el contacto directo con el suelo (duchas) y los estados de inmunosupresión ${ }^{23,31}$, y que la presencia de inflamación periungular $\mathrm{r}^{6,15,30,32}$, así como determinadas presentaciones clínicas ${ }^{6,9,24,32-36}$, permiten sospechar el agente etiológico. Sin embargo, estas afirmaciones se basan en experiencias personales con un escaso número de casos $\mathrm{y}$, por lo tanto, en la actualidad es discutible que las onicomicosis por Fusarium spp. di- 


\begin{tabular}{|c|c|c|c|c|}
\hline Autores & Ciudad & Año & $\begin{array}{l}\text { Número de } \\
\text { aislamientos }\end{array}$ & Especies \\
\hline${ }^{26}$ Vélez H & Medellín & 1988 & 32 & Fusarium spp. \\
\hline${ }^{7}$ Zuluaga A, et al. & Medellín & 2001 & 107 & Fusarium spp. \\
\hline${ }^{27}$ Escobar ML, et al. & Medellín & 2003 & 156 & $\begin{array}{c}\text { Fusarium spp., F. solani, } \\
\text { F. oxysporum, F. verticillioides }\end{array}$ \\
\hline${ }^{58}$ Álvarez MI, et al. & Cali & 2004 & 9 & Fusarium spp. \\
\hline${ }^{28}$ Zuluaga A, et al. & Medellín & 2005 & 591 & Fusarium spp. \\
\hline${ }^{20}$ Castro-López N, et al. & Bogotá & 2009 & 128 & $\begin{array}{l}\text { F. solani, F. oxysporum, } \\
\text { F. verticillioides }\end{array}$ \\
\hline${ }^{59}$ Pérez JE, et al. & Manizales & 2011 & 21 & Fusarium spp. \\
\hline
\end{tabular}

TABLA 1. Onicomicosis por Fusarium en Colombia

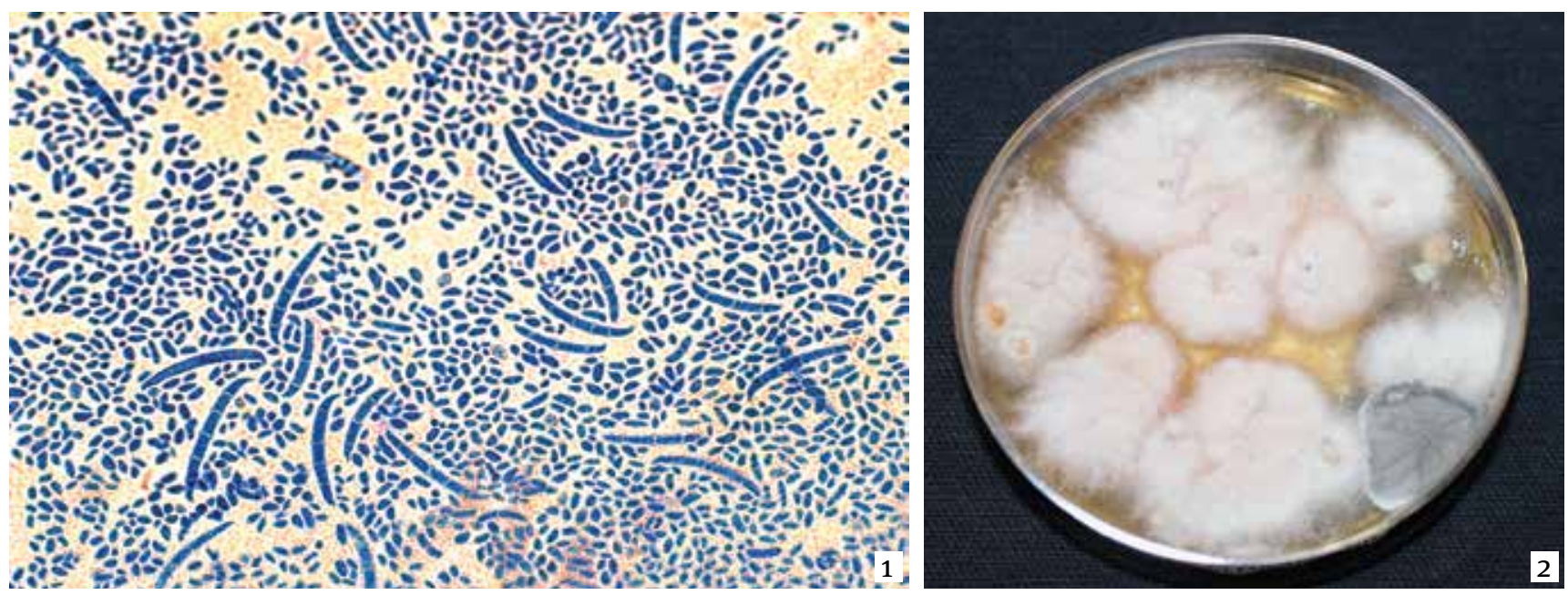

FIGURA 1. Examen microscópico de una colonia de Fusarium spp. Macroconidias de curvatura moderada con hifas tabicadas. Preparación con azul de lactofenol. 40X. Figura 2. Cultivo en medio de Sabouraud. Colonias inicialmente blancas, algodonosas, que se tornan amarillentas y de color naranja-melón. Crecimiento de Fusarium spp. en todos los sitios de siembra.

fieran de las causadas tanto por otros mohos como por dermatofitos, en aspectos tan importantes como su epidemiología y presentación clínica.

El objetivo de este estudio fue describir las características epidemiológicas y clínicas de una población dermatológica con diagnóstico confirmado de onicomicosis por Fusarium spp.

\section{Materiales y métodos}

Se hizo un estudio descriptivo de corte transversal, en el que se describieron las características epidemiológicas y clínicas de los pacientes con diagnóstico confir- mado de onicomicosis por Fusarium spp. en un centro de referencia, entre el $1^{\circ}$ de enero de 2001 y el 31 de diciembre de 2010, con información obtenida a partir de las historias clínicas y las bases de datos del Laboratorio de Micología.

Se incluyeron todos los pacientes que consultaron a este centro durante el periodo establecido, en quienes se aisló Fusarium spp. en dos cultivos a partir de muestras diferentes, utilizando criterios estándar de laboratorio para identificación micológica (FIGURAS 1 Y 2).

Se excluyeron los pacientes con un primer cultivo positivo para Fusarium spp. y una segunda muestra donde se aisló Fusarium spp. y un hongo dermatofito. 


\begin{tabular}{|ccc}
\hline Factor predisponente & $\mathrm{n}$ & $\%$ \\
\hline Contacto con el suelo & 50 & 52,6 \\
\hline Contacto zoonótico & 22 & 23,2 \\
\hline Tinea pedis & 19 & 20 \\
\hline Antecedente de trauma & 13 & 13,6 \\
\hline Uso de piscinas y duchas públicas & 7 & 7,4 \\
\hline Antecedente de pedicure & 3 & 3,2 \\
\hline Práctica deportiva & 1 & 1,0 \\
\hline \hline
\end{tabular}

TABLA 2. Factores predisponentes para onicomicosis por Fusarium spp.

Se hizo un análisis descriptivo de la información mediante el uso de medidas de tendencia central y de dispersión, según los tipos de variables. Se utilizaron los programas Microsoft Office Excel ${ }^{\circledR}$ 97-2003 y Stata ${ }^{\circledR}$ 10.0.

El estudio fue aprobado por el Comité de Ética en Investigación institucional, dando cumplimiento a la legislación nacional e internacional ${ }^{37,38}$. En ningún momento se violó la privacidad de los pacientes ni se hizo ningún tipo de intervención. Según las categorías de riesgo establecidas en la Resolución 8430 de 1993, este estudio fue catalogado como sin riesgo ${ }^{37}$.

\section{Resultados}

Se confirmaron 95 casos de onicomicosis por Fusarium spp. en 72 mujeres y 23 hombres, con una edad promedio de 40 años en ambos sexos; el paciente de menor edad tenía 9 años y el de mayor edad tenía 85 años. El $82 \%$ procedía de áreas urbanas de Bogotá y la mayoría eran empleados de oficina ( $28 \%$ ) y amas de casa ( $16 \%$ ).

El tiempo de evolución promedio fue de 60 meses, con rangos desde 2 hasta 360 meses. El $50 \%$ de los casos tenían menos de 24 meses de evolución.

Entre los factores predisponentes, el más relevante fue el contacto regular con el piso, por el hábito de caminar o tomar duchas sin utilizar calzado, seguido por la convivencia con mascotas (perros, gatos, pájaros ornamentales y conejos); otros factores fueron: la presencia de signos clínicos sugestivos de tinea pedis evidentes durante la consulta; el trauma en las uñas afectadas; el uso de piscinas y duchas públicas; el arreglo de las uñas por el arreglo de uñas por pedicurista y la práctica deportiva (TABLA 2).

El $18 \%$ de los pacientes tenían antecedentes de enfermedades asociadas que podrían favorecer la onicomicosis: insuficiencia vascular de miembros inferiores (8/95), hipotiroidismo (6/95), diabetes mellitus (2/95) y psoriasis (1/95). Sin embargo, ninguno de ellos tenía $\mathrm{VIH}$ o antecedentes de tratamiento con medicamentos inmunosupresores.

No se registraron casos en las uñas de las manos. Predominó el compromiso bilateral de las uñas del primer dedo del pie y solo tres pacientes tenían afectadas las uñas de otros dedos diferentes al primero (TABLA 3).

La presentación clínica más frecuente fue la onicomicosis subungular distal y lateral, evidente en $75,5 \%$ de los casos, seguida de la distrófica total en el 10,6 \%; en $14,5 \%$ no se pudo definir la presentación clínica debido a una descripción deficiente en la historia clínica. Solo un paciente tuvo compromiso proximal (FIGURA 3) y ninguno presentó eritema o inflamación periungular.

Los hallazgos semiológicos que predominaron fueron la onicólisis $(63,1 \%)$, la paquioniquia $(83,1 \%)$ y la cromoniquia (93,6\%). Los colores de la cromoniquia fueron blanco, amarillo, pardo, negro, verde y algunas combinaciones (TABLA 4).

En un caso se confirmó crecimiento de Fusarium spp. y Neoscytalidium dimidiatum a partir de dos muestras repetidas.

\section{Discusión}

Aunque los dermatofitos son la causa de onicomicosis más frecuente en todo el mundo ${ }^{39}$, durante los últimos 15 años se ha informado un incremento en el número de casos de onicomicosis por mohos no dermatofitos $^{8,18}$, entre ellos F. oxysporum, F. solani y Fusarium spp. ${ }^{40,41}$. Se estima que las infecciones por Fusarium spp. representan entre el 1 y el 7,5\% de todas las onicomicosis ${ }^{9,15,18,41}$, pero en Colombia se han informado frecuencia mayores, hasta de $13,8 \%{ }^{28}$.

En contraste con series de casos que tuvieron en

\begin{tabular}{cccccccccc}
\hline \hline $\begin{array}{c}\text { Primer dedo } \\
\text { unilateral }\end{array}$ & \multicolumn{2}{c}{$\begin{array}{c}\text { Primer dedo } \\
\text { bilateral }\end{array}$} & \multicolumn{2}{c}{$\begin{array}{c}\text { Primer dedo } \\
\text { y otros dedos }\end{array}$} & Otros dedos & \multicolumn{2}{c}{ Total } \\
\hline $\mathrm{n}$ & $\%$ & $\mathrm{n}$ & $\%$ & $\mathrm{n}$ & $\%$ & $\mathrm{n}$ & $\%$ & $\mathrm{n}$ & $\%$ \\
39 & 41 & 33 & 34,8 & 20 & 21 & 3 & 3,2 & 95 & 100 \\
\hline
\end{tabular}

TABLA 3. Localización de las lesiones en las uñas de los dedos de los pies. 


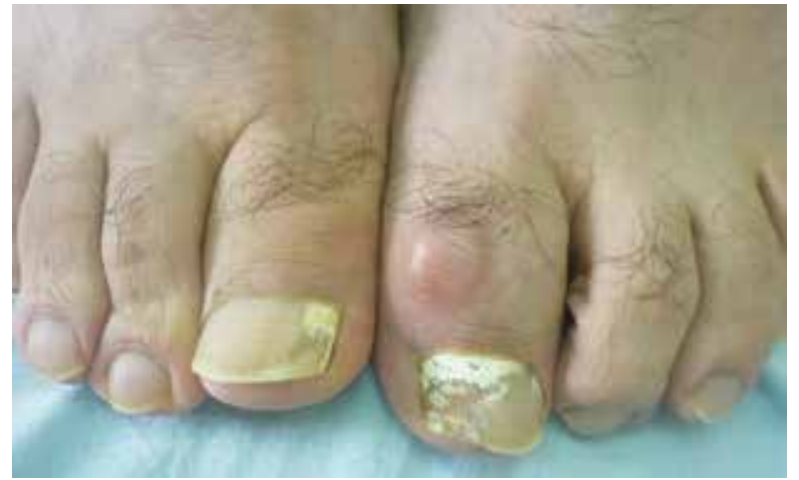

FIGURA 3. Onicomicosis subungular proximal y lateral (mixta) por Fusarium spp.

\begin{tabular}{lll}
\hline Color & $\mathbf{n}$ & $\%$ \\
\hline \hline Amarillo & 31 & 32,7 \\
\hline Blanco-amarillo & 21 & 22,2 \\
\hline Pardo-amarillo & 16 & 16,8 \\
\hline Blanco & 11 & 11,6 \\
\hline Pardo & 3 & 3,2 \\
\hline Verde-amarillo & 3 & 3,2 \\
\hline Blanco-amarillo-pardo & 1 & 1,0 \\
\hline Blanco-amarillo-verde & 1 & 1,0 \\
\hline Pardo-negro & 1 & 1,0 \\
\hline Verde & 1 & 1,0 \\
\hline Sin cromoniquia & 4 & 4,2 \\
\hline Sin registro & 2 & 2,1 \\
\hline Total & 95 & 100 \\
\hline \hline
\end{tabular}

TABLA 4. Diferentes colores de la cromoniquia por Fusarium spp.

cuenta periodos similares ${ }^{27,28}$, la frecuencia de onicomicosis por Fusarium spp. que se informa aquí sugiere que este moho no es un patógeno común en la población que consulta a la institución donde se llevó a cabo el estudio: pacientes provenientes de las áreas urbanas de Bogotá, pues de los 370 casos de onicomicosis que se confirman cada año en el Laboratorio de Micología del Centro Dermatológico Federico Lleras Acosta, solo 2,5 \% son causados por Fusarium spp.

La mayor prevalencia en el sexo femenino de las onicomicosis por Fusarium spp. ya ha sido informada en otros estudios ${ }^{7,15,20,28,41}$, al igual que en las onicomicosis por dermatofitos y levaduras ${ }^{39}$. En nuestra población la relación hombre/mujer fue 1:3,2, lo cual se podría explicar por la mayor preocupación que generan las anormalidades en las uñas entre las mujeres, motivando así la consulta. También, se ha sugerido que el uso de calzado descubierto, más utilizado por las mujeres, favorece el trauma y el contacto directo de las uñas y las plantas con el suelo y, a partir de allí, con el moho saprófito ${ }^{15}$. Sin embargo, el uso de este tipo de calzado no es común en la población urbana de Bogotá.

En general, las onicomicosis por mohos no dermatofitos afectan a los adultos, particularmente entre los 30 y los 50 años $7,15,20,23,30,42$, rango similar al promedio de nuestra población; sin embargo, el $7 \%$ de los casos se presentó en menores de edad, un rango de edad en el que apenas se han informado casos aislados ${ }^{41,43}$ y que no ha sido descrito previamente en la literatura científica nacional. Este último hallazgo y el tiempo de evolución de la enfermedad, a partir de dos meses, y que fue menor de 24 meses en el $50 \%$ de los casos, coincide con la evolución de las onicomicosis por Trichophyton rubrum, Fusarium spp. y $N$. dimidiatum informadas en un estudio con 15 casos, en el que el $46 \%$ tuvo menos de 36 meses de evolución ${ }^{23}$, y contradice la opinión de otros autores que consideran que las onicomicosis por mohos no dermatofitos se caracterizan por largos periodos de evolución, con promedios que varían entre los 3,5 y los 40 años ${ }^{7,20}$. En otra serie con 137 casos de onicomicosis por Fusarium spp., el $79 \%$ de los pacientes tenían entre un mes y cinco años de evolución ${ }^{20}$.

A pesar de que no existen estudios que permitan establecer factores de riesgo específicos para las onicomicosis por mohos no dermatofitos, diferentes autores han considerado como factores predisponentes, especialmente para onicomicosis por Fusarium spp., el trauma y el contacto con el suelo durante oficios como la agricultura y la jardinería ${ }^{18,30}$. El contacto con el suelo, determinado por el hábito de caminar o tomar duchas sin utilizar ningún tipo de calzado, se identificó en un poco más de la mitad de nuestros pacientes $(52,6 \%)$. Lo anterior puede llegar a ser relevante como factor de riesgo para una población procedente de un área urbana, sin antecedentes ocupacionales de contacto con suelos vegetales y plantas. Otros factores, como el contacto zoonótico, el tipo de calzado (sandalias, tenis y botas de caucho), la asistencia a lugares de recreación húmedos (piscinas), el uso común de utensilios para arreglo de uñas, la práctica deportiva y los antecedentes de trauma, mencionados en otros estudios ${ }^{1,20,23,26,27,31}$, no tuvieron relevancia en esta población.

La probable asociación entre onicomicosis por mohos no dermatofitos y enfermedades sistémicas, como aquellas que causan inmunosupresión, se originó a partir del concepto de los mohos como patógenos secundarios ${ }^{3-5,18}$ y ha sido reafirmada en la literatura científica por co- 
mentarios de expertos ${ }^{2}$ e informes de casos de onicomicosis por Fusarium spp. y otros mohos en pacientes neutropénicos, principalmente con $\mathrm{VIH}^{3,31}$, neoplasias hematológicas ${ }^{31,44} \mathrm{y}$ con trasplantes ${ }^{32}$ que han advertido el riesgo de diseminación hematógena potencialmente mortal a partir de estos focos ${ }^{45-48}$. Sin embargo, en esta población de pacientes ambulatorios de un centro de referencia en Dermatología, ningún individuo estaba inmunosuprimido, tal como ha sido informado en otras series de casos de onicomicosis por Fusarium spp. . $^{1,15,30,41}$. En nuestro grupo de estudio, solo 17 casos tenían antecedentes de enfermedad sistémica, principalmente insuficiencia vascular $(8,4 \%)$, lo cual coincide con la asociación entre onicomicosis por mohos no dermatofitos e hipotermia secundaria a enfermedad vascular periférica ya informada en casos de onicomicosis por Scopularopsis brevicaulis ${ }^{49}$. En otros estudios no se ha descrito la asociación entre estas enfermedades sistémicas y las onicomicosis por mohos no dermatofitos ${ }^{23,26,42}$.

La uña del primer dedo del pie se considera la localización más frecuente de onicomicosis, tanto por $\mathrm{Fu}$ sarium spp. ${ }^{1,6,14,18,30}$ como por otros mohos ${ }^{24,26,42,49} \mathrm{y}$ por dermatofitos ${ }^{50}$, tal como se observó en nuestra población, probablemente debido al lento crecimiento de las uñas de los pies, a la mayor exposición y contacto de esta uña con el suelo, a la acumulación de detritos en los pliegues ungulares y al riesgo de trauma, distrofia e infección por dermatofitos ${ }^{6}$, situaciones que pueden ser aprovechadas por los mohos para invadir la lámina ungular ${ }^{34,49,51,52}$. Aunque también se han informado casos de onicomicosis por Fusarium spp. en las uñas de los pulgares ${ }^{53}$, en la población estudiada no se registraron casos de lesiones en las uñas de las manos.

Los cambios ungulares más frecuentes en las onicomicosis por mohos no dermatofitos son la onicólisis, la cromoniquia y la paquioniquia ${ }^{7,15,26}$, hallazgos observados en la mayoría de nuestros pacientes. Solo en cuatro casos no se observó pigmento anormal en las uñas, y el predominio de la cromoniquia de tono amarillento y blanco coincide con los primeros informes de onicomicosis por Fusarium spp., donde se describió como principal hallazgo clínico el compromiso subungular proximal, definido inicialmente como leuconiquia proximal, aislada o adyacente al pliegue ungular ${ }^{34,36}$, presente solo en uno de nuestros pacientes (Figura 3). Teniendo en cuenta lo anterior, algunos autores han considerado esta presentación clínica como característica de las onicomicosis por Fusarium spp..$^{14,54-56}$, principalmente por la especie $F$. oxysporum ${ }^{6,18}$, mientras que otros relacionan este agente etiológico con onicomicosis del tipo subungular proximal que se acompaña de inflamación periungular ${ }^{1,24,48,52,57}$. Sin embargo, el predominio de la forma subungular distal y lateral $(74,7 \%)$ en esta $y$ en otras series de $\operatorname{casos}^{16,31,41,42}$ permite afirmar que las onicomicosis por Fusarium spp. pueden tener diferentes presentaciones clínicas ${ }^{7,32}$, que son indistinguibles de las causadas por otros mohos y por dermatofitos. Por lo anterior, el estudio micológico completo, practicado por especialistas entrenados en la toma y procesamiento de las muestras, es fundamental para la adecuada recuperación e identificación del agente etiológico ante cualquier sospecha clínica de onicomicosis.

\section{Conclusión}

Fusarium spp. es un agente etiológico de onicomicosis por mohos no dermatofitos, aislado con relativa frecuencia en diferentes ciudades de Colombia; sin embargo, se identificó como causa de onicomicosis solo en 95 pacientes en un centro de referencia de Bogotá durante los últimos 10 años, donde se confirman un promedio de 370 casos de onicomicosis al año.

A pesar de que se han descrito características epidemiológicas y clínicas muy particulares, en la población estudiada predominó la forma subungular y lateral, similar a la que presentan las onicomicosis causadas por otros agentes. Por lo tanto, no es posible relacionar determinada presentación clínica con la infección por Fusarium spp.

En este estudio no se encontró relación con ninguno de los factores predisponentes previamente informados en la literatura científica, lo cual evidencia el desconocimiento que aún existe en cuanto a este aspecto de las onicomicosis por mohos no dermatofitos.

\section{Referencias}

1. Baran R, Tosti A, Piraccini B. Uncommon clinical patterns of Fusarium nail infection: Report of three cases. Br J Dermatol. 1997;136:424-7.

2. Hay RJ. Fusarium infections of the skin. Curr Opin Infect Dis. 2007;20:115-7.

3. Moreno G, Arenas R. Other fungi causing onychomycosis. Clin Dermatol. 2010;28:160-3.

4. Ellis DH, Watson A, Marley J, Williams T. Non-dermatophytes in onychomycosis of the toenails. Br J Dermatol. 1997;136:490-3.

5. English MP. Nails and fungi. Br J Dermatol. 1976;94:697-701.

6. van der Straten MR, Balkis MM, Ghannoum MA. The role of nondermatophyte molds in onychomycosis: Diagnosis and treatment. Dermatol Ther. 2002;15:89-98.

7. Zuluaga A, Tabares A, Arango M, Robledo M, Restrepo A, Lotero M. Importancia creciente de los géneros Fusarium y Scytalidium como agentes de onicomicosis. Rev Asoc Colom Dermatol. 2001;9:593-9. 
8. Schechtman RC. Nondermatophytic filamentous fungi infection in South America -Reality or misdiagnosis? Dermatol Clin. 2008;26:271-83.

9. García-Martos P, Domínguez I, Marín P, Linares M, Mir J, Calap J. Onicomicosis por hongos filamentosos no dermatofitos en Cadiz. Enferm Infecc Microbiol Clin. 2000;18:319-24.

10. Gentles J, Evans E. Infection of the feet and nails with Hendersonula toruloidea. Med Mycol. 1970;8:72-5.

11. Campbell C, Mulder J. Skin and nail infection by Scytalidium hyalinum sp. nov. Med Mycol. 1977;15:161-6.

12. Restrepo A, Arango M, Vélez H, Uribe L. The isolation of Botryodiplodia theobromae from a nail lesion. Med Mycol. 1976;14:1-4.

13. López-Jodra O, Torres-Rodríguez JM. Especies fúngicas poco comunes responsables de onicomicosis. Rev Iberoam Micol. 1999;16:11-5.

14. Gugnani HC. Nondermatophytic filamentous keratinophilic fungi and their role in human infection. Rev Iberoam Micol. 2000;17:109-14.

15. Guilhermetti E, Takahachi G, Shinobu CS, Svidzinski TI. Fusarium spp. as agents of onychomycosis in immunocompetent hosts. Int J Dermatol. 2007;46:822-6.

16. Hattori N, Shirai A, Sugiura Y, Li W, Yokoyama K, Misawa Y, et al. Onychomycosis caused by Fusarium proliferatum. Br J Dermatol. 2005;153:647-9.

17. Weitzman I. Saprophytic molds as agents of cutaneous and subcutaneous infection in the immunocompromised host. Arch Dermatol. 1986;122:1161-8.

18. Gianni C, Cerri A, Crosti C. Non-dermatophytic onychomycosis. An understimated entity? A study of 51 cases. Mycoses. 2000;43:29-33.

19. Garcés EG, Orozco MO, Bautista GR, Valencia H. Fusarium oxysporum el hongo que nos falta conocer. Acta Biol Colomb. 2001;6:7-25.

20. Castro-López N, Casas C, Sopó L, Rojas A, Del Portillo P, Cepero MC, et al. Fusarium species detected in onychomycosis in Colombia. Mycoses. 2009;52:350-6.

21. Avendaño C, Arbeláez G, Rondón G. Control biológico del marchitamiento vascular causado por Fusarium oxysporum $f$. sp. phaseoli en fríjol Phaseolus vulgaris L., mediante la acción combinada de Entrophospora colombiana, Trichoderma sp. y Pseudomonas fluorescens. Agron Colomb. 2006;24:62-7.

22. Pérez E, Posada F, González M. Patogenicidad de un aislamiento de Fusarium sp. encontrado infectando la broca del café Hypothenemus hampei. Rev Colom Entomol. 1996;22:105-11.

23. Gómez-Franco J, Navarro-Restrepo B, Restrepo-Botero S, SalazarParra D. Factores de riesgo en adquisición y/o transmisión de onicomicosis por Trichophyton Rubrum, Fusarium spp. y Scytalidium dimidiatum. CIB Medellín 1993-1995. CES Med. 1996;10:17-24.

24. Negroni R, Arechavala A, Bohnvel P. Hongos miceliales no dermatofitos en onicodistrofias. Experiencia de un centro médico privado en Buenos Aires. Dermatol Argent. 2008;14:118-23.

25. Oyeka C, Gugnani H. Keratin degradation by Scytalidium species and Fusarium solani. Mycoses. 1998;41:73-6.

26. Vélez H. Onicomicosis por hongos saprofitos: informe de 49 casos. Iatreia. 1988;1:91-7.

27. Escobar ML, Carmona-Fonseca J. Onicomicosis por hongos ambientales no dermatofíticos. Rev Iberoam Micol. 2003;20:6-10.
28. Zuluaga A, de Bedout C, Tabares A, Cano LE, Restrepo A, Arango $\mathrm{M}$, et al. Comportamiento de los agentes etiológicos de las onicomicosis en un laboratorio de micologia de referencia (Medellín 1994-2003). Med Cutan Iber Lat Am. 2005;33:251-6.

29. Mendoza N, Palacios C, Cardona N, Gómez LM. Onicomicosis: afección común de difícil tratamiento. Rev Asoc Colom Dermatol. 2012;20:149-58.

30. Ranawaka RR, de Silva N, Ragunathan RW. Onychomycosis caused by Fusarium sp in Sri Lanka: Prevalence, clinical features and response to itraconazole pulse therapy in six cases. J Dermatol Treat. 2008;19:308-12.

31. Bonifaz A, Cruz-Aguilar P, Ponce RM. Onychomycosis by molds. Report of 78 cases. Eur J Dermatol. 2007;17:70-2.

32. Gupta AK, Baran R, Summerbell RC. Fusarium infections of the skin. Curr Opin Infect Dis. 2000;13:121.

33. Piraccini BM, Tosti A. White superficial onychomycosis: Epidemiological, clinical, and pathological study of 79 patients. Arch Dermatol. 2004;140:696-701.

34. Rush-Munro F, Black H, Dingley JM. Onychomycosis caused by Fusarium Oxysporum. Aust J Derm. 1971;12:18-29.

35. Salas-Campos I, Hernández-Chavarría F. Fusarium como agente etiológico de onicomicosis: informe de tres casos y revisión de la literatura. Rev Costarric Cienc Méd. 2005;26:53-9.

36. Zaias N. Superficial white onychomycosis. Med Mycol. 1966;5:99.

37. Ministerio de Salud de Colombia. Normas científicas, técnicas y administrativas para la investigación en salud. Resolución $\mathrm{N}^{\mathrm{O}}$ o08430 de 1993. Fecha de consulta: 16 de mayo de 2011. Disponible en: http://www.urosario.edu.co/urosario_files/a2/a24fbo7af561-4fcc-b611-affff4374bb7.pdf.

38. World Medical Association. Declaration of Helsinki: Ethical principles for medical research involving human subjects; 2008. Fecha de consulta: 16 de mayo de 2011. Disponible en: http:// www.wma.net/en/3opublications/1opolicies/b3.

39. Relloso S, Arechavala A, Guelfand L, Maldonado I, Walker L, Agorio I, et al. Onicomicosis: estudio clínico, epidemiológico y micológico multicéntrico. Revista Iberoam Micol. 2012;29:157-63.

40. Gupta A, Elewski B. Nondermatophyte causes of onychomycosis and superficial mycoses. Current Top Med Mycol. 1996;7:87-97.

41. Godoy P, Nunes F, Silva V, Tomimori-Yamashita J, Zaror L, Fischman O. Onychomycosis caused by Fusarium solani and Fusarium oxysporum in São Paulo, Brazil. Mycopathologia. 2004;157:287-90.

42. Hwang SM, Suh MK, Ha GY. Onychomycosis due to nondermatophytic molds. Ann Dermatol. 2012;24:175-80.

43. Romano C, Papini M, Ghilardi A, Gianni C. Onychomycosis in children: A survey of 46 cases. Mycoses. 2005;48:430-7.

44. Boutati EI, Anaissie EJ. Fusarium, a significant emerging pathogen in patients with hematologic malignancy: Ten years' experience at a cancer center and implications for management. Blood. 1997;90:999-1008.

45. Hidalgo I, Garaguso GM, Galimberti G, Galimberti R, Kowalczuk A. Fusariosis diseminada en síndrome linfoproliferativo. Presentación de dos casos. Dermatol Argent. 2008;14:191-5.

46. Nucci M, Anaissie E. Cutaneous infection by Fusarium species in healthy and immunocompromised hosts: Implications for diag nosis and management. Clin Infect Dis. 2002;35:909-20. 
47. Bader M, Jafri AK, Krueger T, Kumar V. Fusarium osteomyelitis of the foot in a patient with diabetes mellitus. Scan J infect Dis. 2003;35:895-7.

48. Tosti A, Piraccini BM, Lorenzi S. Onychomycosis caused by nondermatophytic molds: Clinical features and response to treatment of 59 cases. J Am Acad Dermatol. 2000;42:217-24.

49. Kacalak-Rzepka A, Maleszka R, Turek-Urasinska K, Kempinska A, Rałajczak-Stefanska V, Rozewicka M. Investigations concerning agents influencing the development of onychomycosis caused by Scopulariopsis brevicaulis. Mikol Lek. 2004;11:283-90.

50. Gupta AK, Jain HC, Lynde CW, Watteel GN, Summerbell RC. Prevalence and epidemiology of unsuspected onychomycosis in patients visiting dermatologists' offices in Ontario, Canada: A multicenter survey of 2001 patients. Int J Dermatol. 1997;36:783-7.

51. Andre J, Achten G. Onychomycosis. Int J Dermatol. 1987;26:481-90.

52. Ramani R, Srinivas C, Ramani A, Kumari T, Shivananda P. Molds in onychomycosis. Int J Dermatol. 1993;32:877-8.
53. Ritchie EB, Pinkerton ME. Fusarium Oxysporum infection of thenail: Report of cases. AMA Arch Derm. 1959;79:705-8.

54. Asbati M, Bell Smythe A, Cavallera E. Onicomicosis por hongos no dematofitos: estudio retrospectivo en 4 años. Rev Soc Ven Microbiol. 2002;22:147-52.

55. Di Salvo A, Fickling A. A case of nondermatophytic toe onychomycosis caused by Fusarium oxysporum. Arch Dermatol. 1980;116:699-700.

56. Cavallera E, Asbati M. Onicomicosis por hongos filamentosos no dermatofitos. Dermatol Venez. 2006;44:4-10.

57. Torres-Rodríguez JM, Sellart-Altisent M. Celulitis y onicomicosis proximal de ambos ortejos mayores producida por Fusarium solani. Rev Iberoam Micol. 2006;23:241-4.

58. Álvarez MI, González LA, Castro LA. Onychomycosis in Cali, Colombia. Mycopathologia. 2004;158:181-6.

59. Pérez JE, Cárdenas C, Hoyos AM. Características clínicas, epidemiológicas y microbiológicas de la onicomicosis en un laboratorio de referencia, Manizales (Caldas), 2009. Infect. 2012;15:168-76. 